Research Article

\title{
"Wireline + Wireless" Networking Remote Monitoring Technology for Analysing the Unloading Deformation Characteristics of the Fractured Surrounding Rock Mass Induced by Underground Excavation
}

\author{
Bin Hu, ${ }^{1}$ Jianlong Sheng, ${ }^{1}$ Jing Li $\mathbb{D},{ }^{1}$ Peng-Zhi Pan $\mathbb{D}^{2}{ }^{2}$ Guangquan Zhang, \\ and Zuyang Ye ${ }^{1}$ \\ ${ }^{1}$ School of Resource and Environmental Engineering, Wuhan University of Science and Technology, Wuhan, Hubei 430081, China \\ ${ }^{2}$ State Key Laboratory of Geomechanics and Geotechnical Engineering, Institute of Rock and Soil Mechanics, \\ Chinese Academy of Sciences, Wuhan, Hubei 430071, China \\ Correspondence should be addressed to Jing Li; 1217838280@qq.com and Peng-Zhi Pan; pzpan@whrsm.ac.cn
}

Received 3 June 2019; Revised 16 August 2019; Accepted 28 August 2019; Published 13 October 2019

Academic Editor: Xu Yang

Copyright ( 12019 Bin Hu et al. This is an open access article distributed under the Creative Commons Attribution License, which permits unrestricted use, distribution, and reproduction in any medium, provided the original work is properly cited.

Collapse or large deformation of fractured surrounding rock mass occurs frequently in underground tunnelling and results in many casualties and extensive property damage. This paper proposed a new type of remote telemetry system for monitoring the mechanical responses of underground tunnels during unloading. This system adopted both wired and wireless networking schemes, including a signal collection and transmission subsystem, a management analysis subsystem, and a remote receiving subsystem, in the tunnels. The application of this new approach in a subway tunnel indicated that the complete unloading performance of a surrounding rock mass can be captured in real time and high frequency using this method, recording the deformation of the surrounding rock, the stress in the bolts, and the stress in the shotcrete between the surrounding rock and steel arch. The in situ experimental study also found that deformation of the fractured surrounding rock mass in the Dashizi Tunnel showed a step-like fluctuating growth pattern. Additionally, the mechanical response of the surrounding rock mass during unloading tended to stabilize when the opening face was approximately $35 \mathrm{~m}$ away from the monitoring section, providing new ways to optimize the excavation process and support measure.

\section{Introduction}

A rock mass is a natural system composed of intact rock separated by a fracture network that can influence the physical-mechanical behaviour of the whole rock structure [1-3]. Many mechanical properties of the rock material, such as anisotropic strength, strain-dependent stiffness, bedding direction effect, and time-dependent damage, affect the stability and uncertainty of tunnel structure safety [4-7]. During the underground excavation of tunnels and caverns, instability accidents of the surrounding fractured rock (such as large deformations and collapse) have caused a number of casualties and equipment losses. Thus, understanding the unloading deformation of fractured surrounding rock mass has become one of the current key problems in the construction of underground tunnels [8-12]. At present, underground tunnel safety monitoring, such as converging deformation monitoring of the surrounding rock and anchor stress monitoring, usually adopts the manual on-site reading of data. The current degree of monitoring automation is not high, and the frequency of acquisition is low; therefore, it is difficult to obtain high-frequency monitoring data of the deformation and stability evolution of the fractured surrounding rock mass. Additionally, when danger signs such as collapse or rock burst occur in tunnels, manual measurements cannot be continued due to personal safety considerations. 
In the past 10 years, the leap forward in optics, electricity, computer technology, and wireless network technology has greatly promoted the research and application of wireless telemetry in geotechnical engineering for further understanding the mechanical response of fractured surrounding rock mass. For example, Ohbayashi et al. [13] adopted a distributed node network system with automatic control, restoration, and efficient transmission functions to monitor the deformation of a landslide body. Although some innovative monitoring attempts have been made, they have obvious shortcomings compared to mature applications applied in this field of study. Ji [14] verified the feasibility of a new displacement monitoring method and the stability of a wireless sensor network for fractured rock masses in underground structures. Li et al. [15] tested the feasibility of the performance and measurement accuracy of a wireless sensor network in a tunnel. Lin [16] established a bus remote topology microcomputer remote system in the Rainbow Ridge Highway Tunnel. These technical developments and application attempts initially show that remote wireless telemetry is feasible in underground engineering, but the multisensor compatible monitoring method and corresponding analysis for the fractured rock mass induced by tunnelling are not yet sufficient.

Thus, this article first proposed a "wireline + wireless" networking automatic monitoring method, which is based on a focus control system (FCS) and General Packet Radio Service (GPRS), for fractured surrounding rock mass induced by underground excavation. Then, the mechanical performance of fractured surrounding rock mass during unloading, including the unloading deformation, anchor stress, and spray pressure, is summarized according to the monitoring data by the multivariable smart sensors in tunnel engineering. The in situ experimental study indicated that the deformation of the fractured surrounding rock mass in Dashizi Tunnel showed a step-like fluctuating growth pattern in the overall trend of change, and the mechanical response of the unloading surrounding rock mass tended to be stable when the opening face was approximately $35 \mathrm{~m}$ away from the monitoring section, providing new information and motivating new mitigation measures for excavation and support optimization.

\section{Remote Monitoring Networking Principle}

Based on the advancement of monitoring data information and the characteristics of underground tunnel engineering, a "wireline + wireless" networking scheme was used in the tunnel to propose a new remote online telemetry system architecture and method, mainly including an information acquisition and transmission subsystem, a management and analysis subsystem, and a remote receiving subsystem for monitoring data (Figure 1). For the dynamic data collection of the site, the information acquisition and transmission subsystem uses the powerful capabilities of signal measurement, data processing, and storage of the intelligent sensor to complete the real-time, on-site collection of raw data of the target object, including the deep displacement, stress, and spray-layer pressure of the surrounding rock

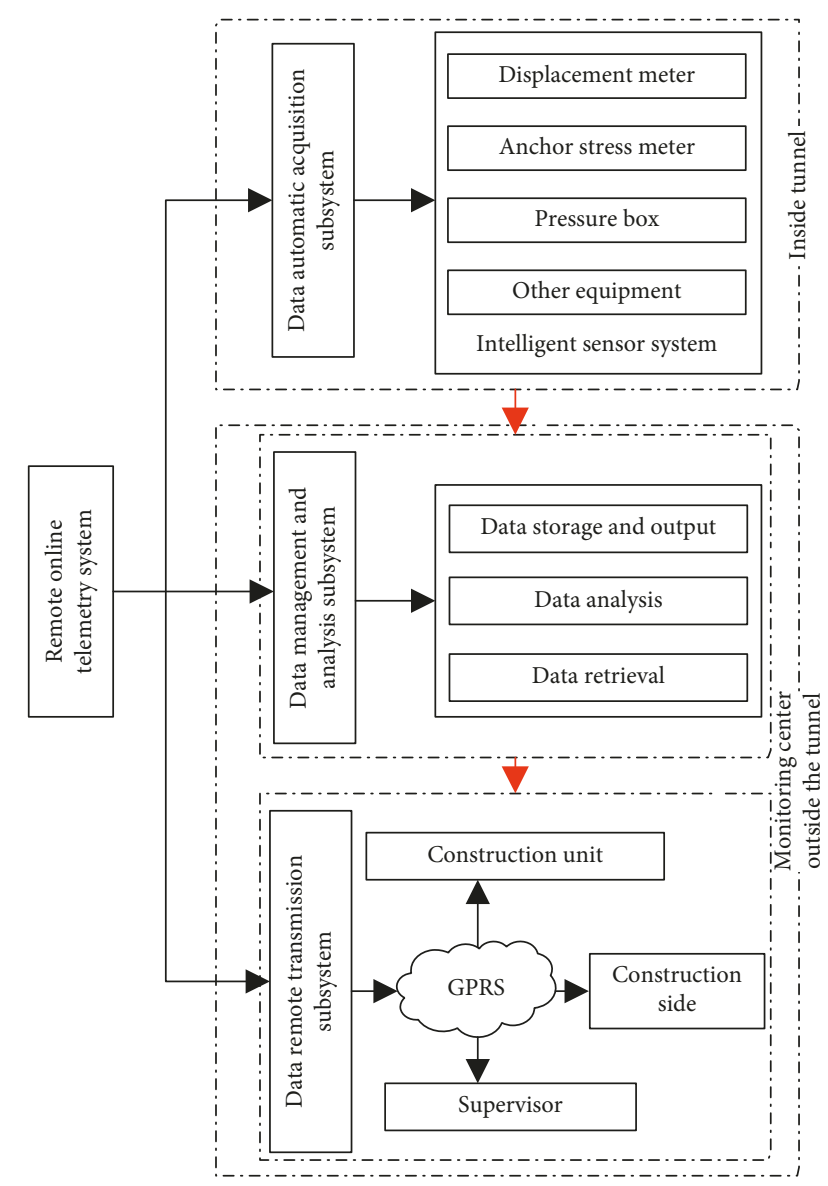

FIgURE 1: Configuration of the remote automatic monitoring system.

material. The management and analysis subsystem manages all field devices by issuing instructions that are mainly responsible for collecting, summarizing, and forwarding data information. The remote receiving subsystem uses GPRS network technology, dynamic domain name resolution technology, and port mapping technology to realize the remote transmission and reception of data information between nonpublic Internet Protocol (IP) networks and to provide the major equipment required for the on-site monitoring centre; this equipment includes monitoring hosts, power supply equipment, and wireless transmission modules. This method effectively solves the difficult problem of the incompatible use of different brands of monitoring equipment on-site while realizing the complete dispersion of the structural control functions and improves the robustness of the system.

2.1. Signal Acquisition Principle. According to the definition of the Institute of Electrical and Electronics Engineers (IEEE), smart sensors are integrated sensors that typically reduce the amount of controlled or perceived data and are applied to the network environment, whose main functions are signal sensing, conditioning, and signal processing and data communication [17]. Compared with traditional sensors, smart sensors have significant advantages in functional processing and can realize the all-digital output of measurement values. 
The terminal collector used in this article contains three kinds of smart sensors: displacement meter, anchor stress meter, and pressure box. Each smart sensor has a unique instrument number. The acquisition system, which is a monitoring host outside the tunnel, reads the sensor data by sending special instructions with the instrument number information to the terminal collector. When the instructions contain a smart sensor's instrument number, the instrument begins to directly transmit the collected data. Due to space limitations, this article uses the displacement meter as an example to briefly introduce its working principle; the structural profile of the displacement meter is shown in Figure 2. The displacement meter is mainly composed of a body, an anchor head, and an extension rod and is based on the principle of an LC oscillation circuit, as shown in the following formula:

$$
f=\frac{1}{2 \pi \sqrt{L C}}
$$

where $f$ is the oscillation frequency and $L$ and $C$ are the inductance and capacitance, respectively.

As shown in Figure 2, the spiral coil, the soft magnetic core, the integrated circuit, and the resonant capacitor combine to form an LC oscillation circuit. The magnetic core moves in the spiral coil, causing the coil inductance $L$ to change as the output signal frequency of the oscillation circuit changes. When the material properties, process technology, and structural form are selected properly, the output signal frequency and inductance exhibit a strong linear relationship $[18,19]$. By measuring the change in coil inductance, a nonelectrical measurement can be realized with an effective range of $200 \mathrm{~mm}$ and a resolution of $0.1 \mathrm{~mm}$.

\subsection{Signal Transmission Principle. The signal transmission} includes the following two parts: the field transmission and the signal remote transmission. The field transmission uses the RS-485 bus architecture, one of the most widely used industrial buses in the industry, with a maximum transmission rate of $10 \mathrm{MB} / \mathrm{s}$ and a maximum distance of $1.2 \mathrm{~km}$. This bus can realize real-time communication of the digital, bidirectional transmission and multibranch structure of onsite intelligent devices and automation systems [20, 21]. The master-slave structure is adopted in the communication transmission. The slaves in the monitoring room outside the tunnel control the slaves in the tunnel (acquisition module), and each slave has unique address information. When the host needs to read the data in the tunnel for acquisition or storage, the host sends a read command. If the address information in the command is the same as the slave address information, the host receives the data. In the transmission process, the slave cannot send the command to the master actively, and the slaves do not send commands to each other to avoid the mutual interference of the slaves in the data reading process. Simultaneously, the serial ports of the slave and host will be set to ensure the same format and communication baud rate. The transmission at the construction site is wired transmission. The signal is first transmitted through the sensor in the form of digital signals in the cable, and then the cable enters the repeater passing through the 4channel acquisition device. The signal is amplified and transmitted from the bus to the information acquisition module in the monitoring room because the repeater compensates for signal attenuation and is finally connected to the monitoring centre server through the USB to the 485 data line to complete the entire field acquisition and transmission, as shown in Figure 3. Due to the adoption of a unified communication protocol and network architecture, this networking mode is fully compatible with different types of sensors, and there are no restrictions on the number and location of access sensors.

Signal transmission utilizes network communication. A new communication network is established by the construction site monitoring centre computer, wireless acquisition module, remote host PC (server), and remote PC (client). Based on the GPRS, domain name resolution, and port mapping technology, the task of remotely sending, transmitting, and receiving one-to-one and one-to-many arbitrary terminals for on-site collection of data is realized. The specific working principle is as follows (Figure 4):

(1) Based on the existing network communication protocol (i.e., the TCP/IP protocol suite), the site monitoring computer is used to complete the serial port setting of the monitoring room GPRS acquisition module, including a Domain Name System (DNS) server address, communication port, and network domain name (acquired by dynamic domain name resolution software).

(2) The IP address, communication port, and network domain name of the host PC (server) are synchronized with the GPRS sending module; simultaneously, dynamic domain name resolution (DNSR) and port mapping technologies are used to complete IP fixing and port number assigning.

(3) The lower machine (client) communication parameters are synchronized with the host computer (server).

(4) The SIM card embedded in the field wireless acquisition module is activated, the Internet network is entered through TCP/IP dial-up, communication with the host computer (server) network is established, and the data transmission phase is entered. In the communication phase, the baud rates of the upper computer and the lower computer are always the same. The main purpose of using domain name resolution and port mapping technology in this approach is to fix the IP address and solve the problem of the nonpublic IPs not being able to communicate with each other.

2.3. Monitoring Network Feature. Using the linear networking model of wired + remote GPRS in the tunnel based on the FCS theory, the openness of the monitoring system and the complete dispersion of the structural functions have been realized. The digital data signal transmission has significantly improved the signal accuracy, and its characteristics are shown as follows: 


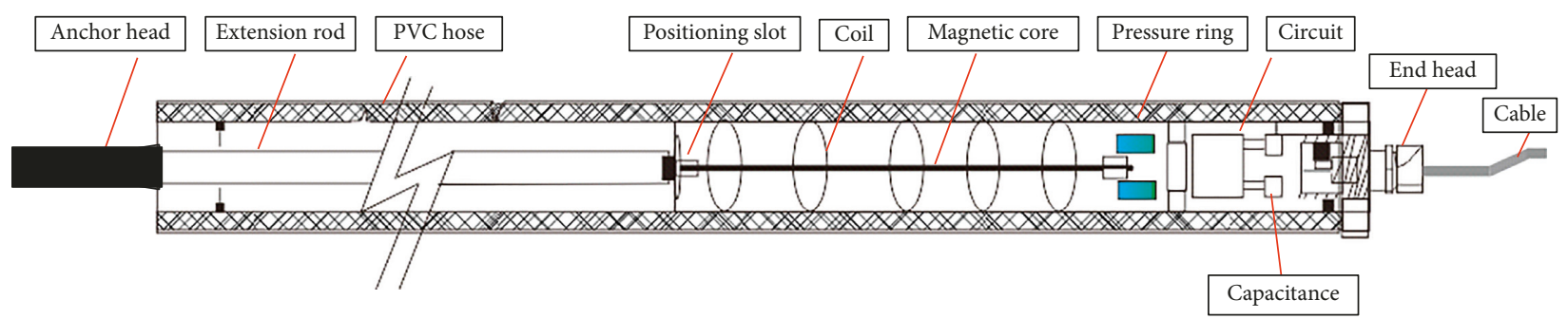

Figure 2: Structural cross section of the displacement meter.

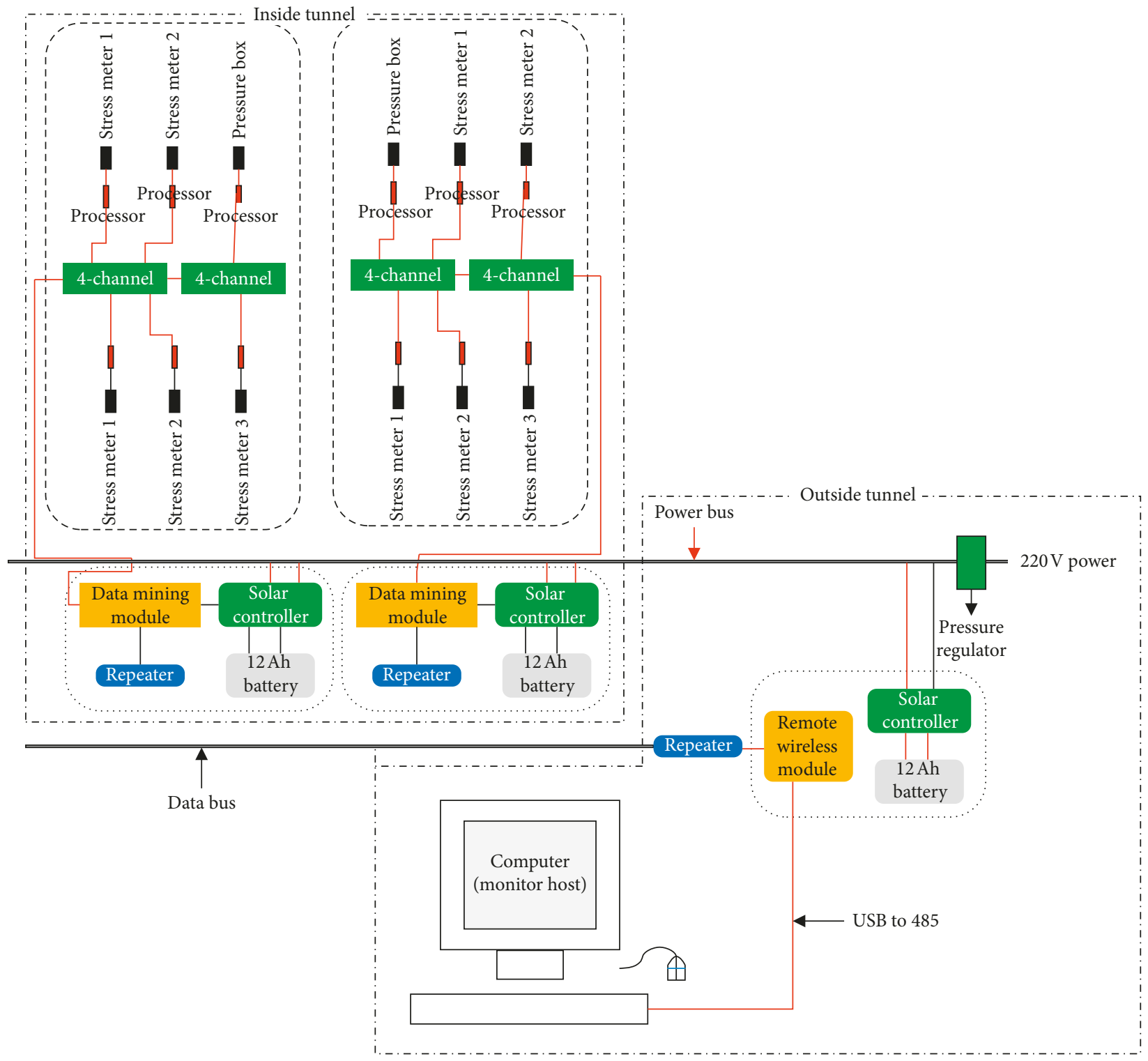

FIGURE 3: Schematic of wired network inside tunnel + wireless network and data transmission.

(1) Instrument number uniqueness: all on-site monitoring instrument numbers are automatically generated by the sensor's built-in chip, and this number is used as the unique identifier for reading and setting the instrument data, thereby eliminating the occurrence of artificial number confusion or information loss.
(2) Good openness and operability: by using the global fifth-generation control system Fieldbus Control System and adopting a unified communication protocol and network structure, the problem that different brands of instruments cannot be combined in low cost in the traditional monitoring system is effectively solved. On this basis, integration and 


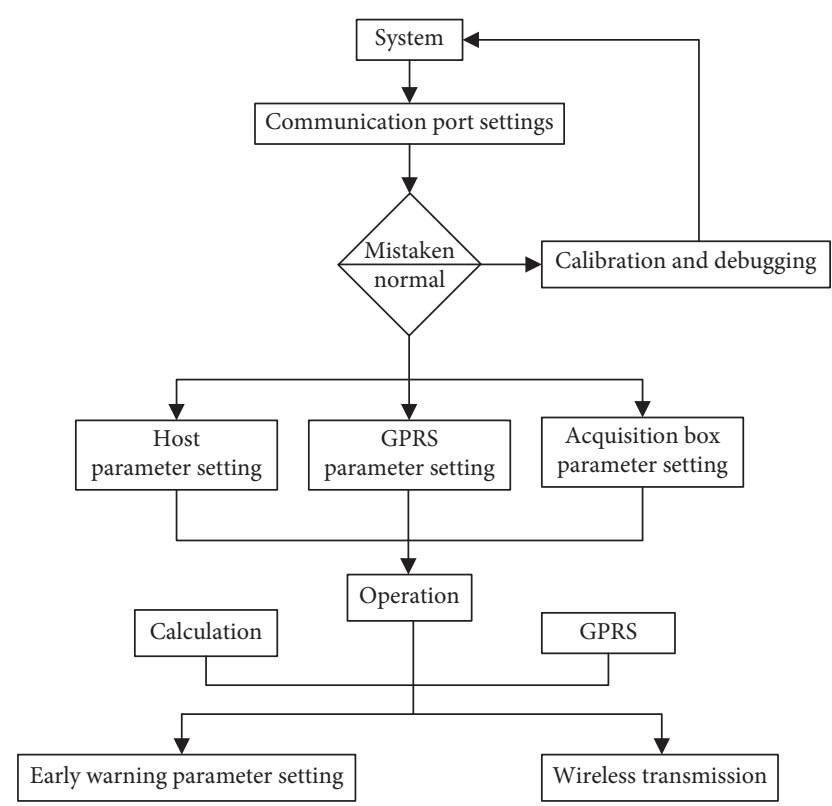

FIgURE 4: Schematic of the program operation.

configuration of different brands of instruments are realized. When a certain instrument fails, the project site can be immediately replaced with other brands of instruments, greatly improving the compatibility and scalability of field device systems.

(3) Functional dispersion mode: by using the "workstation-field smart meter" secondary organizational structure instead of the original distributed control system (DCS) input/output unit and control station mode, the original control station function is distributed to the field smart meters, forming a virtual control station.

(4) Long-distance digital transmission: all smart sensors in the monitoring site have a built-in calculation chip. When they collect data, they will automatically convert the measurement data and directly output the digital physical quantity. The output signal accuracy is improved nearly 10 -fold over the current 4-20 mA DC analogue signal, and a transmission distance of up to $1.2 \mathrm{~km}$ can be achieved without distortion without adding additional repeaters.

\section{Information regarding the Dashizi Tunnel and Monitoring Method}

3.1. Project Overview. The Sanmen Bridge and the connection project in Zhejiang Province started from Daigang, Xiangshan, and Ningbo and were connected to the end of the south terminal of the Xiangshan Bridge. There are seven tunnels along the line. The Dashizi Tunnel is the longest tunnel in this project, with a total length of $3882.5 \mathrm{~m}$ and a two-cave, 4-lane layout, making it the longest road tunnel in Ningbo.

The tunnel passes through hilly terrain with steep mountains. Two stations (ZK50 + 032 and YK50 + 028) span the diversion tunnel of the Longxi'an-Cang'ao Reservoir with a small difference in height. Bedrock joints and fissures are developed in this rock, and the integrity is average. According to geological surveys, the entire tunnel has four fragmentation belts with a total length of approximately $260 \mathrm{~m}$. The weathering at both tunnel openings is severe, with developed joints and fractures; the joint-intensive areas are partially visible. The integrity near the rock interface is poor, with a weak self-stabilizing performance, and local collapse instability occurred during construction. Regarding the surrounding rock in the tunnel, type III accounts for $60.7 \%$ of the total length and types IV and V account for approximately $1165 \mathrm{~m}$, or approximately $40 \%$ of the total length. Due to abundant summer rain in southeastern Zhejiang Province, the characteristics of the tuff tunnels that undergo water softening are obvious. Joint fissures occur in the surrounding rock that is not type III, and the unloading free surface formed by tunnel excavation is large. Therefore, the risk of large deformation and collapse of the surrounding rock during excavation is high, and it is necessary to systematically conduct remote monitoring of the safety of the surrounding rock, as shown in Figure 5.

3.2. Monitoring Section Instrument Embedding Method. When monitoring the stability of the surrounding rock within the tunnel, the on-site intelligent sensor location selection, the initial value check, and a correct installation are directly related to the success or failure of the overall monitoring work.

3.2.1. Embedded Monitoring Instrument Design. According to the characteristics of the external deformation sensitivity of landslides, the monitoring indicators include deformation, anchor stress, and spray pressure. In principle, each monitoring section within the system has 3 quickdisplacement meters, 2 anchor stress gauges, and 1 spraylayer pressure gauge embedded within it (Figure 6). One fast-installed displacement meter is embedded at each random monitoring point.

3.2.2. Displacement Meter Installation and Burial. To reduce the working hours within the tunnel, the displacement meter structure was assembled off-site in advance. When burying the instruments, the assembled displacement meter was first sent into the cave to be drilled, while the brackets were assembled at the bottom of the remaining displacement meters outside the cave. Then, the tray was welded and fixed to the I-steel frame. Finally, the grouting was completed.

3.2.3. Anchor Stress Gauge Installation and Burial. The welding part and the body at both ends of the anchor stress gauge are thread-connected. Before the assembly, the welding part is taken out to the site for welding. The installation procedure at the site is the same as the displacement meter.

3.2.4. Pressure Box Installation and Burial. Due to the undulating surface of the dome, the pressure box is difficult to fit completely and force evenly. Therefore, a flattened 


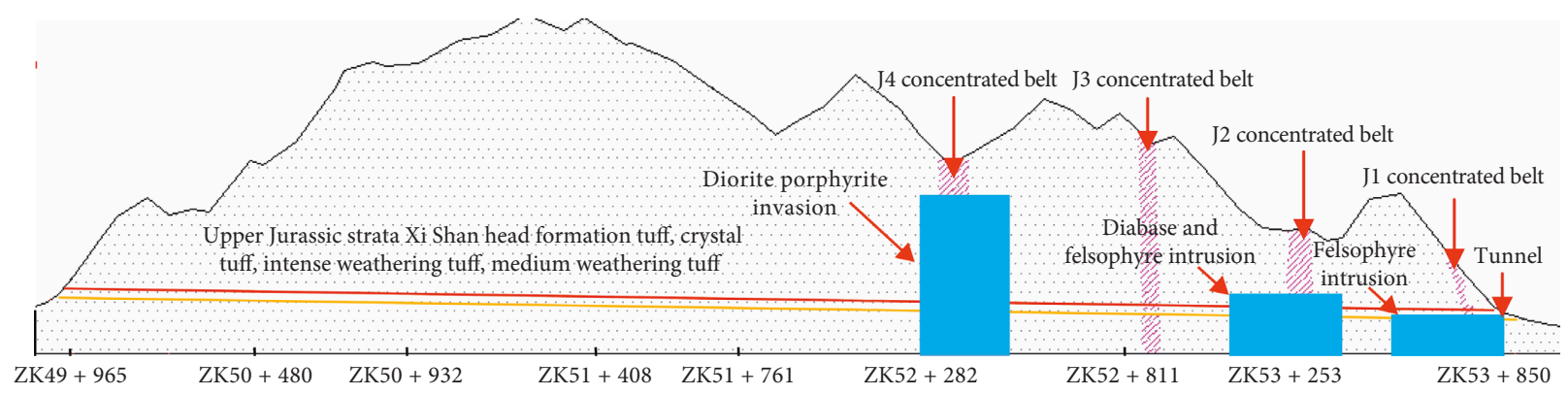

FIGURE 5: Geological longitudinal section of the Dashizi Tunnel and classification of the surrounding rock rupture zone.

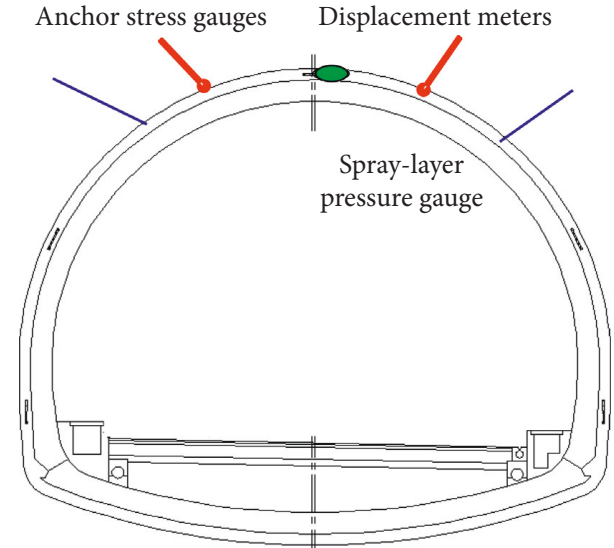

Figure 6: Section showing the arrangement of the instruments.

sandbag with a slightly larger area than the surface area of the pressure box must be prepared in advance. This sandbag is placed to be in contact with the surface of the rock surrounding the vault at the top of the pressure box, and the bottom is lifted using a homemade balanced steel pallet. The tray and I-beam are welded and fixed in the process of embedding on-site.

3.3. Site Layout Example. To realize weather monitoring of the surrounding rock deformation and supporting structure under the condition of no outside WiFi signal, a "wired + wireless" networking scheme was adopted. The tunnel adopts cable-based signal acquisition and transmission. After the signal is concentrated in the cave, it is connected to the on-site wireless transmission module and then enters the Internet through GPRS transmission. Finally, remote data processing software is used to read and process the prewarning data.

3.3.1. Station Layout Design. Prior to the monitoring work, the high-risk caverns identified in the "Dashizi Tunnel Safety Risk Analysis" report were taken into consideration. Following this report, combined with the engineering geological exploration report and the numerical analysis of the tunnel stability, a number of system monitoring sections and multiple random monitoring points were set up in the tunnels with high risk of collapse. For example, in the monitoring section of the opening section and the tunnel section, monitoring sections are mainly located at $Z K 52+840$ to $Z K 53+260$ and ZK53 + 638 to ZK53 + 840 .

3.3.2. Wireless Signal Network Transmission. On-site wireless signal networking mainly considers the high efficiency and stability of data transmission that is prominently reflected in 7 aspects: the distributed access, bus control, integration, GPRS transmission, remote acquisition, automatic analysis, and directional distribution. In the process of slag removal and secondary lining, the machinery is likely to cause damage to the line during the construction process, affecting the data transmission because of the poor light and the limited visual field in the cave. As the depth of the tunnel excavation increases, the intensity of the wireless signal in the tunnel and the strength of the signal gradually weaken as the transmission distance increases. In this regard, it is determined that wired transmission and remote GPRS wireless transmission are both used from the inside of the tunnel to the monitoring room. Considering the narrow characteristics of the tunnel, a conventional mesh network mode is selected instead of a linear network for the slope; a conventional mesh network mode can increase the effective transmission distance and can be more economical. In addition, in order to reduce the number of traces in the tunnel, two buses (power and signals) are drawn from the monitoring room and are located on the side of the fire cavern. Bus control is implemented, and the collection box is connected in series with the bus to avoid excessive use of the instrument line. The information transmission and power supply are realized by this means, which will not only facilitate the transmission but also reduce the cable length. In this project, the buses are arranged on the right side, as shown in Figure 7. The field wireless signals from all monitoring instruments at each monitoring section transmit through the connected 4-channel collectors via cables and then are transmitted to the tunnel acquisition module and repeater (repeater connected to bus) through a 4channel acquisition device; finally, they are transmitted remotely through the wireless transmitter module (Figure 8).

3.4. Site Layout Example. At present, the determination of the domestic safety coefficient of surrounding rock is mainly based on two important criteria, the tunnel displacement and empirical value of the plastic zone, but there is considerable controversy. For the tunnel warning parameters, domestic regulations do not provide intuitive and universal 


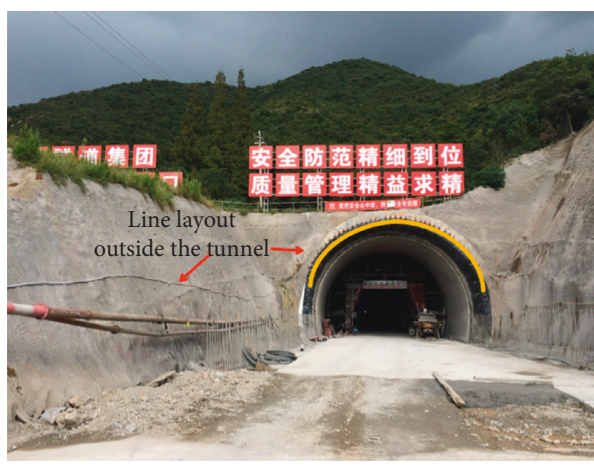

(a)

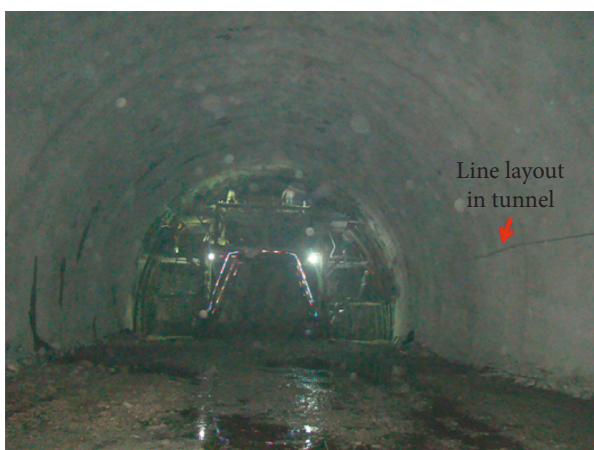

(b)

Figure 7: Layout of the field monitoring system: (a) line layout outside the tunnel; (b) line layout in the tunnel.

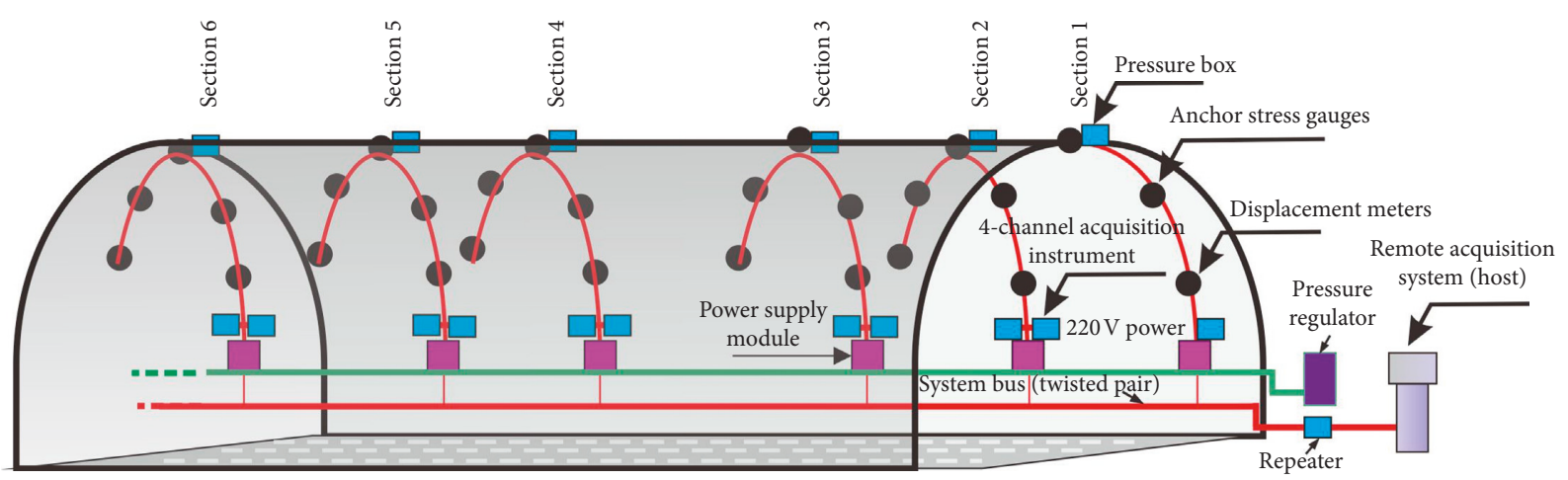

FIgURE 8: Network transmission route inside the tunnel.

quantitative regulations. Therefore, safety factors are mostly adopted in design, and the calculation of the limit displacement is emphasized. The limit displacement is considered to be a combination of theory and practice and can reflect the actual situation to some extent, as discussed [22-25]. Here, we proposed the statistical analysis of on-site displacement monitoring data as a criterion for the stability of the surrounding rock; this criterion relies on the statistical analysis of monitoring data and combines equations (2) and (3) to classify the monitoring and warning levels of the left tunnel of the Dashizi Tunnel into orange and red level II, indicated as warnings and alarms, respectively. In addition, sending objects and levels are set in the warning software system, and warning information is issued in a targeted manner.

$$
\begin{gathered}
U_{\mathrm{a}}=\frac{D_{\mathrm{m}}}{L_{\mathrm{s}}}, \\
V=\frac{D_{\mathrm{m}}}{t},
\end{gathered}
$$

where $U_{\mathrm{a}}$ is the relative value of the allowable displacement of the surrounding rock mass, $D_{\mathrm{m}}$ is the cumulative measured value of the displacement between two points of the surrounding rock mass, $L_{\mathrm{m}}$ is the distance between the two measuring points on the surrounding rock mass (the actual depth of burial of the instrument, a fixed value of $3.6 \mathrm{~m}$ ), and the statistically derived $t$ is 26 days. Based on the strength reduction method, $U_{\text {a }}$ of the tunnel is calculated to be $0.5 \%$. Substituting $L_{\mathrm{s}}$ and $U_{\mathrm{a}}$ into equation (2) gives an alarm value of $18 \mathrm{~mm}$; additionally, the warning value is statistically calculated to be $10 \mathrm{~mm}$. Finally, the alarm values and warning values are substituted into equation (2) to obtain deformation rates of $0.38 \mathrm{~mm} / \mathrm{d}$ and $0.69 \mathrm{~mm} / \mathrm{d}$, respectively.

\section{Result Analysis and Discussion}

Since the tunnel remote monitoring team entered the site to carry out monitoring work in July 2015, a large amount of monitoring data have been collected. Due to space limitations and the prewarning value setting discussed in the previous section, this article focuses on the analysis of typical monitoring data of only some sections.

4.1. Displacement Analysis. Comparing Figures 9 and 10, we can see that there is a certain relationship between the deformation and stabilization time of the surrounding rock material and the geological conditions. It shows that the worse the geological condition, the greater the subsidence of the surrounding rock dome and the longer the time required for stabilization. The section of ZK53 +782 is in the Grade V surrounding rock section, with joint fractures and poor integrity. The deformation rate of the dome increases rapidly at the beginning of excavation. The deformation of the arch 


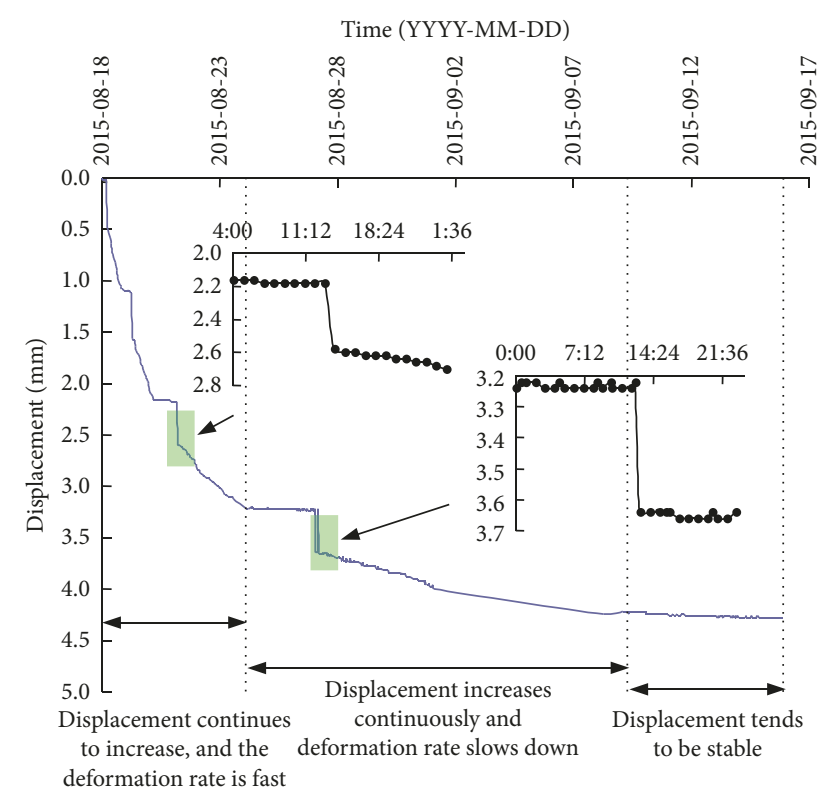

FIGURE 9: Displacement-time curves of the surrounding rock at the cross section of ZK53 +782 .

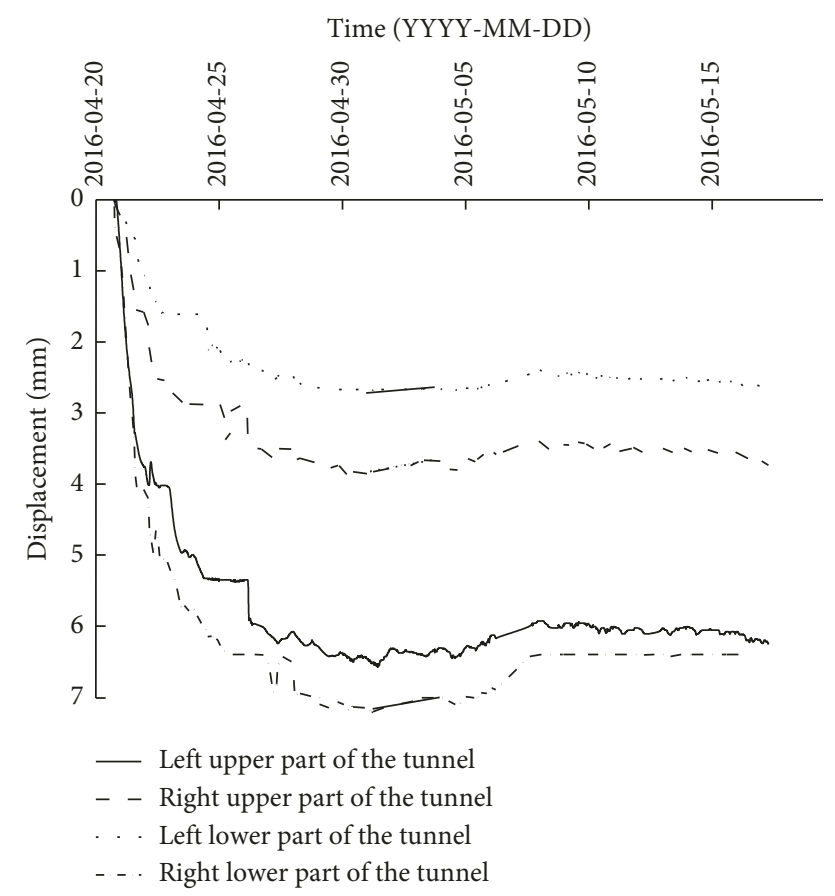

FIGURE 10: Displacement-time curves of the surrounding rock at the cross section of ZK52+888.

support decreases after approximately 20 days, then continues to decrease slowly, and stabilizes after 26 days. The section of ZK52 +888 is Grade IV surrounding rock. The rate of subsidence decreases after 12 days of shotcretebolting support and tends to stabilize after 18 days. In addition, the blasting excavation work in the later period has an effect on the stability of the surrounding rock mass in the early period. The curves show that the displacements of the section laid in the first 5 to 7 days after the blasting significantly fluctuate. Moreover, the small graphs in the figures indicate that some instrument values are subject to sudden change due to shocks. According to the data statistics, when the distance between the monitoring section and the working surface increases to approximately $30 \mathrm{~m}$, the excavation disturbance effect is significantly reduced, and the monitoring displacement curve shows a step-like fluctuation growth pattern throughout the process. The process of monitoring displacement curve as they change rapidly in the early stage, slowly in the middle stage, and gradually stabilize in the later stage is basically consistent with the conclusion drawn from the analysis of similar tunnels in the later stage of domestic monitoring [26].

4.2. Stress Analysis. An anchor stress meter was buried in the wall on both sides of the tunnel at a depth of $3.6 \mathrm{~m}$. In addition to resisting the inward deformation of the surrounding rock by adding steel arches and shotcrete, the anchor rod also bears the pulling force generated by the later deformation of the surrounding rock mass itself. It shows that the overall trend of stress development is similar to the aforementioned displacement change rule. The stress value in the early period of measurement shows a rapid increase, and it falls after a month or so. Additionally, the corresponding stress growth rate gradually decreases and then tends to stabilize (Figure 11). Compared with the related domestic tunnel stress monitoring results, the peak level of this stress measurement is not high. This information suggests that the anchor rod mainly plays a synergistic and auxiliary supporting role to the deformation of the surrounding rock, while the steel arch and surrounding rock self-supporting mechanism play the main supporting roles [27].

4.3. Pressure Analysis of the Steel Arch. The pressure box is placed between the initial steel arch and the surrounding rock. Figure 12 shows the pressure-time curve of the initial support of the surrounding rock. This figure shows that the change in the compressive stress is sensitive and that the rapid growth period is mainly concentrated in the first 5 days but subsequently stabilized as the face excavation continued.

4.4. Discussion. Based on the analysis of displacement, stress, and steel arch pressure, it is found that the system can effectively realize the monitoring and early warning of tunnel excavation deformation. This paper mainly analyses the monitoring system and its monitoring data without further discussion. The factors affecting the deformation of rock mass of fractures in underground excavation are complicated. How to combine monitoring with project risk analysis is one of the difficult problems to be solved urgently. In the last decade, the probabilistic approaches which can integrate the sources of uncertainties with geotechnical reliability analysis have been well developed [28, 29]. In order to better analyse the deformation characteristics of fractured rock mass in underground excavation project, we 


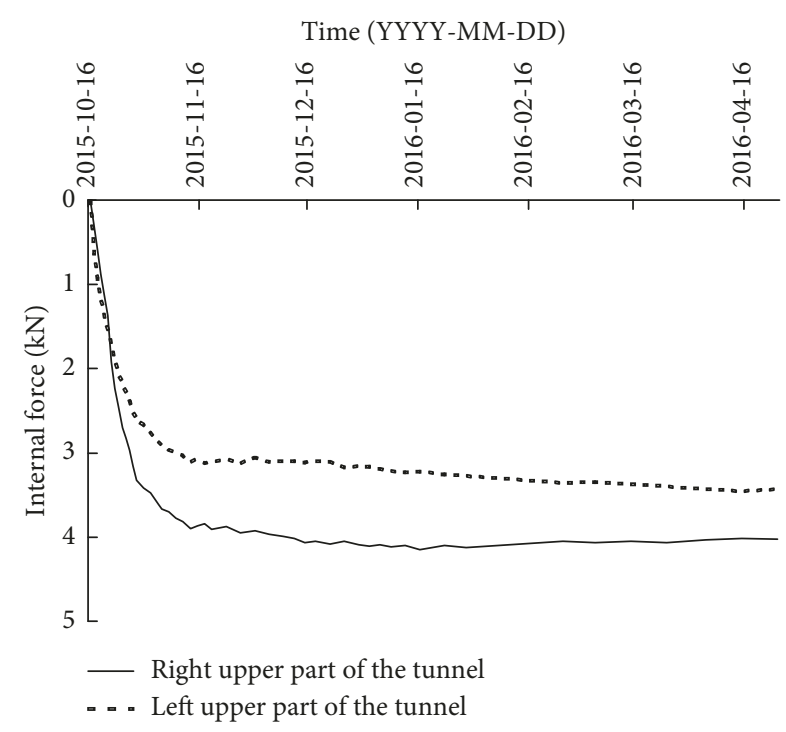

Figure 11: Internal stress-time curves of the surrounding rock at the cross section of ZK52+560.

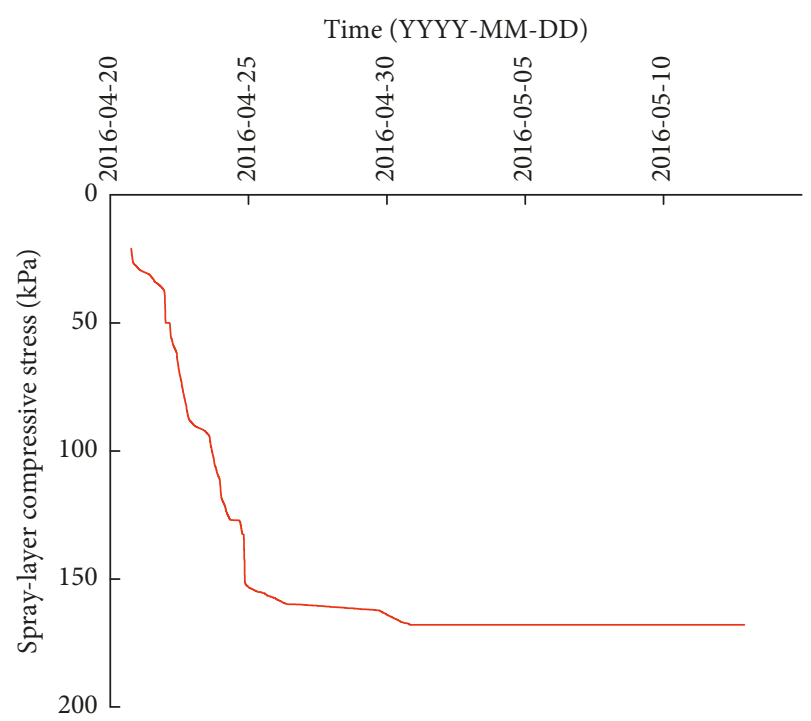

Figure 12: Spray pressure stress-time curve of the surrounding rock at the cross section of ZK52 +560 .

plan to conduct further research from the perspective of monitoring and probability method.

\section{Conclusion}

Based on the FCS theory and GPRS packet wireless transmission technology, a new tunnel remote online telemetry system construction method was proposed for monitoring the unloading deformation characteristics of fractured surrounding rock mass due to underground excavation. This method effectively solves the difficult problem of the incompatible use of different brands of monitoring equipment on-site while realizing the complete dispersion of the structural control functions, improving the robustness of the system.
Through analysis of the stress-time and displacementtime curves obtained by high-frequency monitoring, it was found that the deformation of the surrounding rock of the Dashizi Tunnel showed an overall step-like fluctuation growth pattern. Because the deformation is prone to abrupt change after blasting, the variation in the deformation and spray pressure was greatly affected by the subsequent tunnel excavation disturbance. However, the deformation, anchor stress, and spray pressure underwent rapid changes in the early period, slow changes in the middle period, and gradual stabilization in the later period. After statistical analysis of all the monitoring data, it was found that the mechanical response of the surrounding rock tended to stabilize when the face was approximately $35 \mathrm{~m}$ away from the buried section (i.e., approximately 2.5 times the diameter of the cave).

\section{Data Availability}

The data used to support the findings of this study are available from the corresponding author upon request.

\section{Conflicts of Interest}

The authors declare no conflicts of interest.

\section{Acknowledgments}

This study was partially funded by the National Natural Science Foundation of China (nos. U1802243 and 41672317), Hubei Province Technical Innovation Special (Major Projects) (no. 2017ACA184), Key Research Program of Frontier Sciences, Chinese Academy of Sciences (grant no. QYZDB-SSW-DQC029), and Major Science and Technology Projects of WUST Cultivate Innovation Teams (no. 2018TDX01). The authors would like to thank Professor Lv Y. G. from CCCC Highway Consultants Co., for the field support.

\section{References}

[1] B. H. G. Brady and E. T. Brown, Rock Mechanics for Underground Mining, Chapman \& Hall, London, UK, 1994.

[2] R. E. Goodman, Introduction to Rock Mechanics, Wiley, New York, NY, USA, 2nd edition, 1989.

[3] S. Miao, P.-Z. Pan, Z. Wu, S. Li, and S. Zhao, "Fracture analysis of sandstone with a single filled flaw under uniaxial compression," Engineering Fracture Mechanics, vol. 204, pp. 319-343, 2018.

[4] X. Z. Wu, "Quantifying the non-normality of shear strength of geomaterials," European Journal of Environmental and Civil Engineering, vol. 22, no. 3, pp. 1-27, 2018.

[5] X. L. Lü, J. G. Qian, and M. S. Huang, "Instability of sands under axisymmetric proportional strain and stress loadings," European Journal of Environmental and Civil Engineering, vol. 23, no. 11, pp. 1294-1310, 2019.

[6] H. Zhou, H. Liu, D. Hu, F. Zhang, F. Yang, and J. Lu, "Estimation of the effective thermal properties of cracked rocks," European Journal of Environmental and Civil Engineering, vol. 20, no. 8, pp. 954-970, 2016.

[7] W. Wang, Y. Cao, Q. Zhu, W. Xu, and J. Shao, "Experimental investigation and constitutive modelling of creep-damage 
behaviours in monzogranite," European Journal of Environmental and Civil Engineering, vol. 19, no. 1, pp. s54-s69, 2015.

[8] X. L. Yang and F. Huang, "Collapse mechanism of shallow tunnel based on nonlinear Hoek-Brown failure criterion," Tunnelling and Underground Space Technology, vol. 26, no. 6, pp. 686-691, 2011.

[9] Q. Jiang, X.-t. Feng, Y. Fan et al., "In situ experimental investigation of basalt spalling in a large underground powerhouse cavern," Tunnelling and Underground Space Technology, vol. 68, pp. 82-94, 2017.

[10] P. Shi, D. Zhang, J. Pan, and W. Liu, "Geological investigation and tunnel excavation aspects of the weakness zones of Xiang'an subsea tunnels in China," Rock Mechanics and Rock Engineering, vol. 49, no. 12, pp. 4853-4867, 2016.

[11] Q. Jiang, X.-t. Feng, Y. H. Hatzor, X.-j. Hao, and S.-j. Li, "Mechanical anisotropy of columnar jointed basalts: an example from the Baihetan hydropower station, China," Engineering Geology, vol. 175, pp. 35-45, 2014.

[12] N.-H. Tran, D.-P. Do, and D. Hoxha, "A closed-form hydromechanical solution for deep tunnels in elastic anisotropic rock," European Journal of Environmental and Civil Engineering, vol. 22, no. 12, pp. 1429-1445, 2017.

[13] R. Ohbayashi, Y. Nakajima, H. Nishikado, and S. Takayama, "Monitoring system for landslide disaster by wireless sensing node network," in Proceedings of the 2008 SICE Annual Conference, pp. 1704-1710, IEEE, Chofu, Japan, August 2008.

[14] Z. Ji, "Application of wireless sensor network technology in the tunnel deformation and disaster monitoring," Tunnel Construction, vol. 34, no. 2, pp. 134-139, 2014.

[15] X. Li, Z. Ji, H. Zhu, and C. Gu, "A feasibility study of the measuring accuracy and capability of wireless sensor networks in tunnel monitoring," Frontiers of Structural and Civil Engineering, vol. 6, no. 2, pp. 111-120, 2012.

[16] S. X. Lin, "Monitoring system design of Caihongling tunnel," Journal of Guangdong Communications Polytechnic, vol. 4, no. 1 , pp. $35-40,2005$.

[17] IEEE Standards Association, IEEE Standard for a Smart Transducer Interface for Sensors and Actuators-Transducer to Microprocessor Communication Protocols and Transducer Electronic Data Sheet (TEDS) Formats, IEEE Standards Association, Piscataway, NJ, USA, 1997.

[18] S. Tumanski, "Induction coil sensors-a review," Measurement Science and Technology, vol. 18, no. 3, pp. R31-R46, 2007.

[19] L. Ben-Brahim and A. Kawamura, "A fully digitized fieldoriented controlled induction motor drive using only current sensors," IEEE Transactions on Industrial Electronics, vol. 39, no. 3, pp. 241-249, 1992.

[20] G. Schickhuber and O. McCarthy, "Distributed fieldbus and control network systems," Computing \& Control Engineering Journal, vol. 8, no. 1, pp. 21-32, 1997.

[21] M. Castro, R. Sebastian, F. Yeves, and J. Peire, "Well-known serial buses for distributed control of backup power plants. RS485 versus controller area network (CAN) solutions," in IEEE 2002 28th Annual Conference of the Industrial Electronics Society, vol. 3, pp. 2381-2386, Sevilla, Spain, November 2002.

[22] X. H. Li, H. T. Wang, J. Q. Jia, C. H. Yang, G. Z. Hu, and Z. X. Xue, "Ultimate displacement discrimination of stability and reliability analysis of surrounding rocks of tunnel and underground engineering," Rock and Soil Mechanics, vol. 26, no. 6, pp. 850-854, 2005.

[23] Q. Jiang, X.-T. Feng, T.-B. Xiang, and G.-S. Su, "Rockburst characteristics and numerical simulation based on a new energy index: a case study of a tunnel at 2,500 m depth,"
Bulletin of Engineering Geology and the Environment, vol. 69, no. 3, pp. 381-388, 2010.

[24] Y. R. Zheng, "The application of FEM limit analysis in tunnel engineering," Journal of Chongqing Jiaotong University (Natural Science), vol. 30, pp. 1127-1137, 2011.

[25] Q. J. Wu, M. N. Wang, and D. G. Liu, "Research on stability of tunnel surrounding rocks based on statistical analysis of onsite displacement monitoring data," Rock and Soil Mechanics, vol. 33, no. 2, pp. 359-364, 2012.

[26] C. C. Xia, J. W. Gong, Y. Tang, and H. H. Zhu, "Study on site monitoring of large-section highway tunnels with small clear spacing," Chinese Journal of Rock Mechanics and Engineering, vol. 26, no. 1, pp. 44-50, 2007.

[27] Q. S. Liu, S. Y. Bai, C. X. Xiao, and W. Gao, "Study on the surrounding rock stability of Longtan tunnel based on in-situ monitoring measurements," Chinese Journal of Rock Mechanics and Engineering, vol. 26, no. 10, pp. 1982-1990, 2007.

[28] J. Ji, C. Zhang, Y. Gao, and J. Kodikara, "Effect of 2D spatial variability on slope reliability: a simplified FORM analysis," Geoscience Frontiers, vol. 9, no. 6, pp. 1631-1638, 2018.

[29] J. Ji, C. Zhang, Y. Gao, and J. Kodikara, "Reliability-based design for geotechnical engineering: an inverse FORM approach for practice," Computers and Geotechnics, vol. 111, pp. 22-29, 2019. 


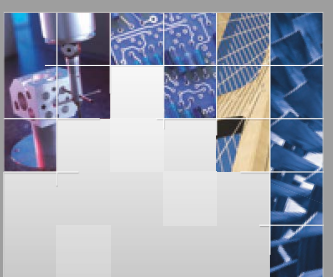

\section{Enfincering}
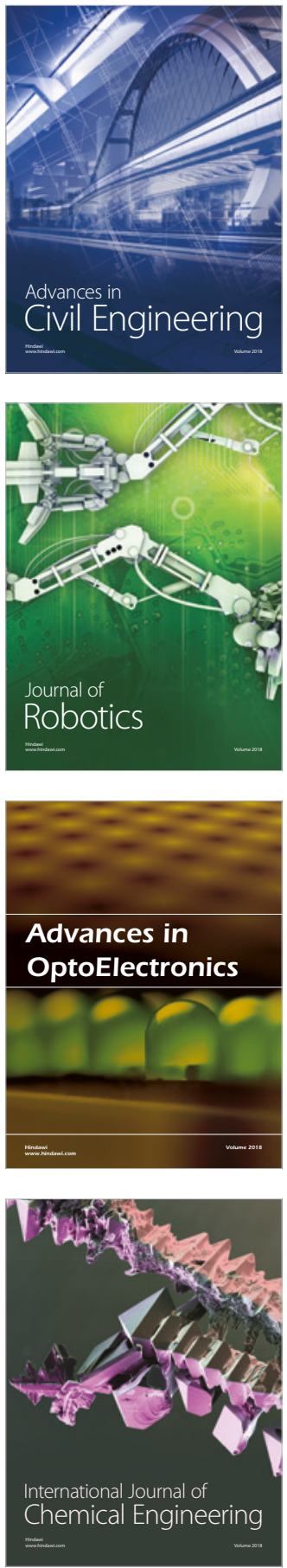

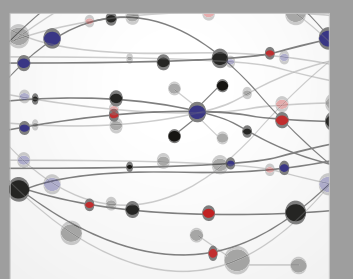

\section{Rotating \\ Machinery}

The Scientific World Journal

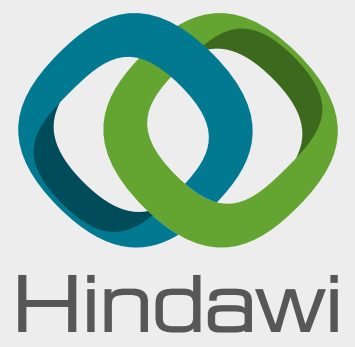

Submit your manuscripts at

www.hindawi.com
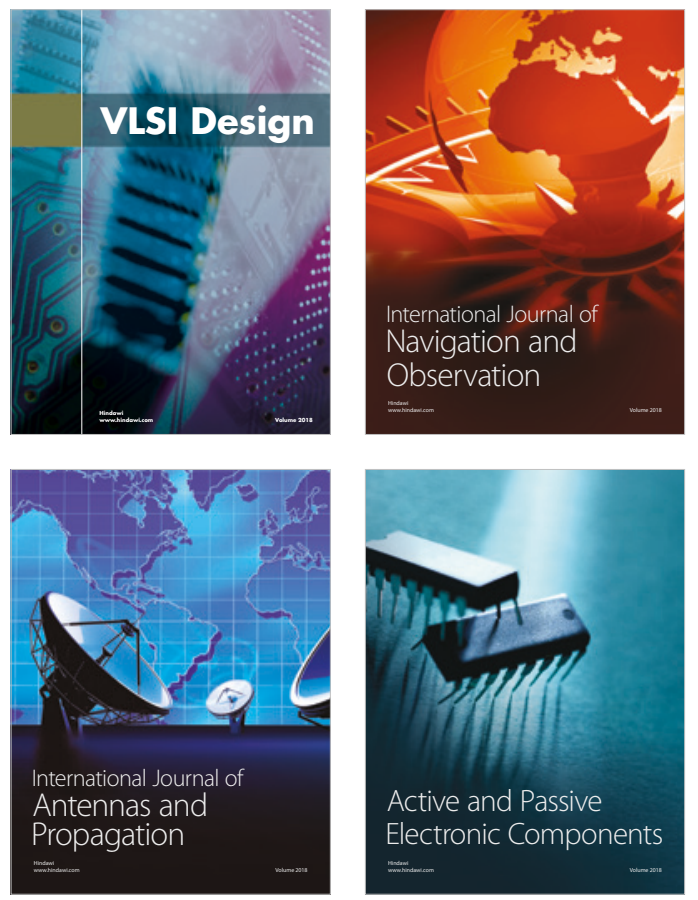
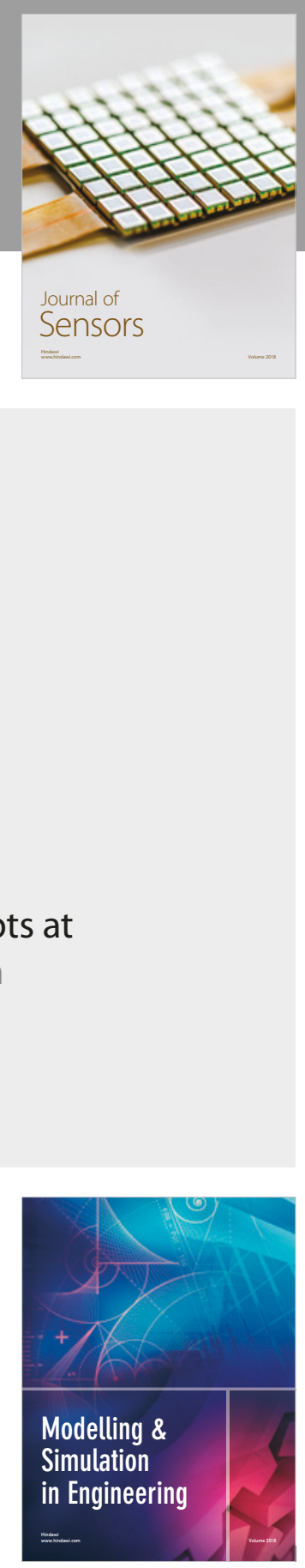

\section{Advances \\ Multimedia}
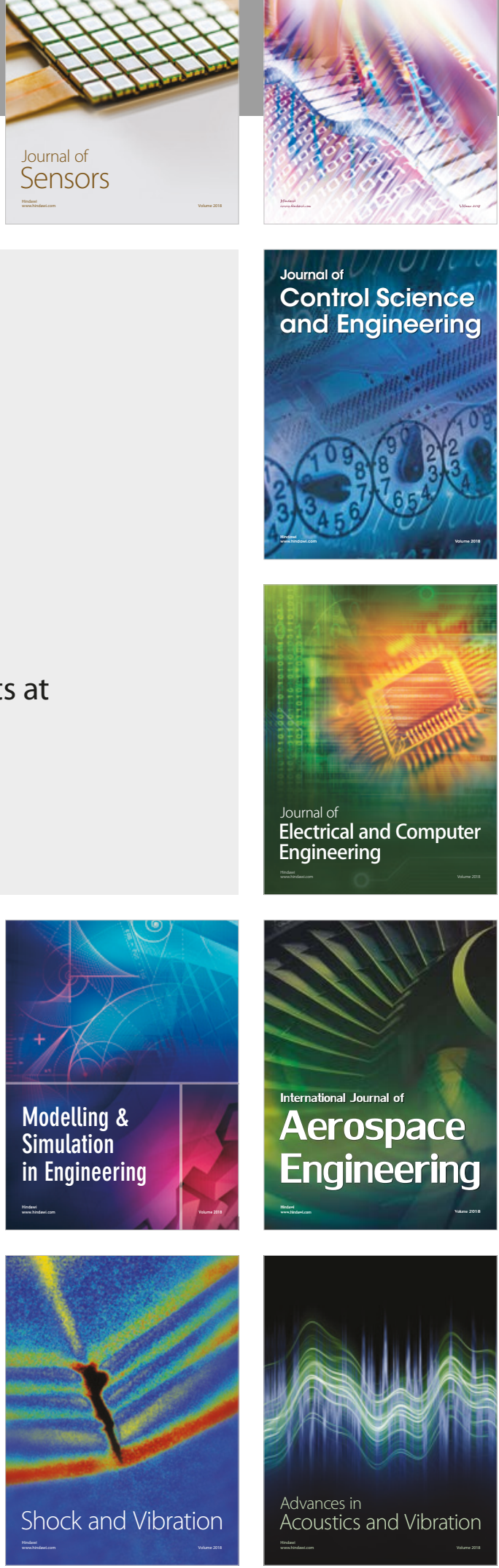